Supporting Information

\title{
Salicylaldimine-Aluminum Complexes for the Facile and Efficient Ring-Opening Polymerization of $\varepsilon$-Caprolactone
}

N. Nomura, T. Aoyama, R. Ishii, and T. Kondo

\section{Generals.}

All manipulations were carried out using standard Schlenk-line techniques. ${ }^{1} \mathrm{H}$ NMR spectra of organic compounds were recorded on Varian Gemini-300 (300 MHz) or Bruker Avance 400 (400 $\mathrm{MHz})$ in $\mathrm{CDCl}_{3}$ or $\mathrm{C}_{6} \mathrm{D}_{6} \cdot{ }^{1} \mathrm{H}(400 \mathrm{MHz})\left(\mathrm{C}_{6} \mathrm{D}_{5} \mathrm{CD}_{3}\right)$ and ${ }^{13} \mathrm{C}(100 \mathrm{MHz})\left(\mathrm{C}_{6} \mathrm{D}_{6}\right) \mathrm{NMR}$ spectra of aluminum complexes were recorded on Bruker Avance 400. ${ }^{27} \mathrm{Al}$ NMR spectra were recorded on JEOL A-600 (156 MHz) in $\mathrm{C}_{6} \mathrm{D}_{5} \mathrm{CD}_{3}$. $\mathrm{N}_{2}$ was purified through a drycolumn ( $T_{\mathrm{d}}$ (dew point) $\leq-80{ }^{\circ} \mathrm{C}$; NIKKA $\mathrm{SEIKO})$ and a gas-clean column $\left(\mathrm{O}_{2} \leq 0.002 \mathrm{ppm}\right.$; NIKKA SEIKO). Molecular weights of the polymers were estimated by size exclusion chromatography (SEC) using polystyrene standards $\left(\mathrm{CHCl}_{3}\right)$. The absolute weight-average molecular weight $\left(M_{\mathrm{w}}\right)$ were obtained using low-angle laser light scattering (TOSOH LS8000).

\section{Materials.}

Toluene was distilled from $\mathrm{Na}$-bezophenone under purified $\mathrm{N}_{2}$ prior to use. $\varepsilon$-Caparolactone was purchased from TCI and was distilled twice under reduced pressure ( 10 torr) after dried over powdery $\mathrm{CaH}_{2}$ over a few days. $\mathrm{Et}_{3} \mathrm{Al}$ in toluene solution $(\sim 1 \mathrm{M})$ was purchased from Kanto Chemicals, and $0.10 \mathrm{M} \mathrm{Et}_{3} \mathrm{Al}$ solution was prepared by dilution of this solution with freshly distilled toluene, and was stored under $\mathrm{N}_{2}$ at $\mathrm{rt}$.

\section{Ring-opening reaction of CL using diphenyl-substituted homosalen complex.}

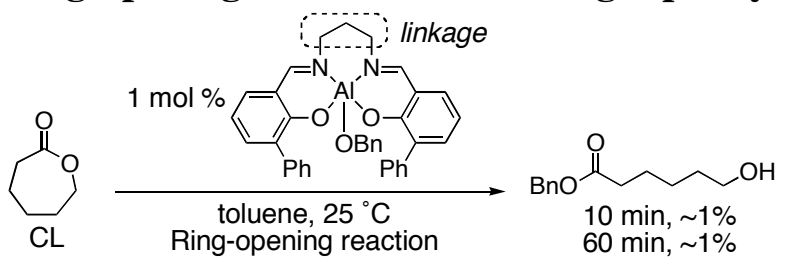

\section{Representative ligand synthesis.}

(1) $N$-(2-hydroxy-3,5-dimethylbenzylidene)-2,4,6-tri-tert-butylaniline (entry 12 in Table 1).

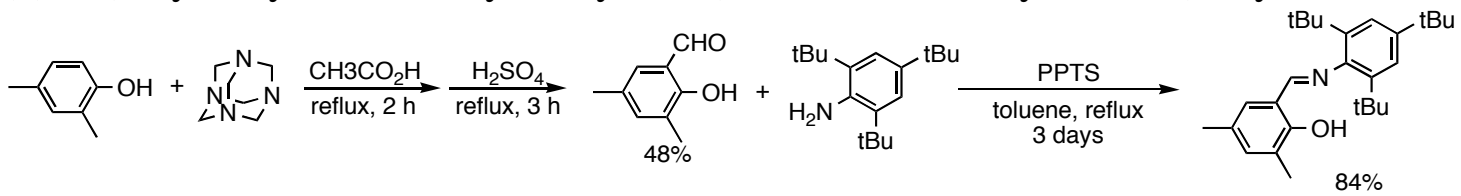

The mixture of hexamethylenetetramine (14 g, $100 \mathrm{mmol}), 2,4$-dimethylphenol $(6.0 \mathrm{~mL}, 50$ $\mathrm{mmol})$, acetic acid $(24 \mathrm{~mL})$ in a flask was heated at $70{ }^{\circ} \mathrm{C}$. After the mixture turned into a solution, it was refluxed at $120-130{ }^{\circ} \mathrm{C}$ for $2 \mathrm{~h}$. It was then cooed at $70{ }^{\circ} \mathrm{C}$, and $33 \% \mathrm{H}_{2} \mathrm{SO}_{4}(24 \mathrm{~mL})$ was added. The mixture was heated at $110{ }^{\circ} \mathrm{C}$ for $3 \mathrm{~h}$. The product was extracted with ether four times $(50 \mathrm{~mL} \times 4)$, and the combined etheral layer was washed with $\mathrm{H}_{2} \mathrm{O}$ four times. The ethereal solution was dried over $\mathrm{MgSO}_{4}$, the crude product was purified by flash column chromatography to afford 2-hydroxy-3,5dimethylbenzaldehyde (3.6 g, 48\%).

To the mixture of 2,4,6-tri-tert-butylaniline (3.0 g, $12 \mathrm{mmol}), p$-toluenesulfonic acid (a 
catalytic amount) and 2-hydroxy-3,5-dimethylbenzaldehyde (3.6 g, $24 \mathrm{mmol})$ was added toluene (10 $\mathrm{mL}$ ). The mixture was heated at reflux for 3 days. Toluene was evaporated in vacuo and excess salicylic aldehyde was mostly removed by Kugelrohr distillation. The residue was recrystallized in EtOH to afford $3.8 \mathrm{~g}$ of the corresponding slicylaldimine $(9.8 \mathrm{mmol}, 84 \%)$.

${ }^{1} \mathrm{H}$ NMR $\left(400 \mathrm{MHz}, \mathrm{C}_{6} \mathrm{D}_{6}\right): \delta 13.50(\mathrm{~s}, 1 \mathrm{H}, \mathrm{Ar}-\mathrm{OH}), 7.97(\mathrm{~s}, 1 \mathrm{H}, \mathrm{ArCH}=\mathrm{NAr}$ ') $7.56(\mathrm{~s}, 2 \mathrm{H}$, $\left.\mathrm{N}-\mathrm{Ar}-\mathrm{H}_{\text {meta }} \times 2\right), 6.85\left(\mathrm{~s}, 1 \mathrm{H}, \mathrm{OAr}-\mathrm{H}_{\text {meta }}\right), 6.69\left(\mathrm{~d}, J=1.6 \mathrm{~Hz}, \mathrm{OAr}-\mathrm{H}_{\text {meta }}\right), 2.31\left(\mathrm{~s}, 3 \mathrm{H}, \mathrm{Ar}-\mathrm{CH}_{3}\right), 2.06$ (s, $\left.3 \mathrm{H}, \mathrm{Ar}-\mathrm{CH}_{3}\right), 1.39$ (s, 18H, NAr-C $\left(\mathrm{C}_{3}\right)_{3}$ ortho $\left.\times 2\right), 1.38$ (s, $\left.9 \mathrm{H}, \mathrm{NAr}-\mathrm{C}\left(\mathrm{C}_{3}\right)_{3 \text { para }}\right)$.

${ }^{13} \mathrm{C}$ NMR (100 MHz, $\left.\mathrm{C}_{6} \mathrm{D}_{6}\right): 168.8(-\mathrm{CH}=\mathrm{N}-), 158.1$ (quaternary C), 148.5 (quaternary $\mathrm{C}$ ), 145.9 (quaternary C), 140.6 (quaternary C), $135.9\left(\mathrm{O}-\mathrm{Ar}_{\text {meta }}\right), 130.0\left(\mathrm{O}-\mathrm{Ar}_{\text {meta }}\right), 127.7$ (quaternary $\left.\mathrm{C}\right), 126.5$ (quaternary C), $122.2\left(\mathrm{~N}-\mathrm{Ar}_{\text {meta }} \times 2\right), 117.5$ (quaternary $\left.\mathrm{C}\right), 36.1\left(\mathrm{~N}-\mathrm{Ar}_{\text {ortho }}-\mathrm{CMe}_{3} \mathrm{X}\right.$ 2), 35.0 $\left(\mathrm{N}-\mathrm{Ar}_{\text {para }}-\underline{\mathrm{C}} \mathrm{Me}_{3}\right), 32.3\left(\mathrm{~N}-\mathrm{Ar}_{\text {ortho }}-\mathrm{C}\left(\underline{\mathrm{C}} \mathrm{H}_{3}\right)_{3} \times 2\right), 31.8\left(\mathrm{~N}-\mathrm{Ar} \mathrm{p}_{\text {para }}-\mathrm{C}\left(\mathrm{CH}_{3}\right)_{3}\right), 20.3\left(\mathrm{Ar}-\mathrm{CH}_{3}\right), 15.6$ $\left(\mathrm{Ar}-\mathrm{CH}_{3}\right)$.

(2) $o$-Formylation of $o$-cresol as a representative of $p$-unsubstituted phenol.

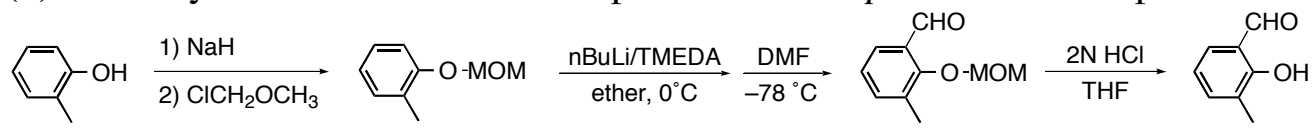

To a suspension of $60 \% \mathrm{NaH}$ in oil $(546 \mathrm{mg})$ in $10 \mathrm{~mL}$ of THF was added $o$-cresol $(1.05 \mathrm{~mL}$, $10.2 \mathrm{mmol}$ ) at $0{ }^{\circ} \mathrm{C}$ under $\mathrm{N}_{2}$. The resulting mixture was stirred for $1 \mathrm{~h}$. Chloromethyl methyl ether (MOM-Cl, $1.30 \mathrm{~mL}, 17.1 \mathrm{mmol}$ ) was then added. After the mixture was stirred for $3 \mathrm{~h}$ at $0{ }^{\circ} \mathrm{C}$, the reaction was quenched with sat. $\mathrm{NH}_{4} \mathrm{Cl}$. The product was extracted with $\mathrm{Et}_{2} \mathrm{O}$. The crude product was purified by flash column chromatography, and the corresponding MOM ether $(1.10 \mathrm{~g}, 71 \%)$ was obtained.

To the MOM ether $(1.10 \mathrm{~g}, 7.22 \mathrm{mmol})$ in $\operatorname{dry}_{2} \mathrm{Et}_{2} \mathrm{O}(15 \mathrm{~mL})$ were added $N, N, N^{\prime}, N^{\prime}-$ tetramethylethylenediamine (TMEDA, $2.2 \mathrm{~mL}, 12.8 \mathrm{mmol})$ and then $\mathrm{nBuLi}(8.0 \mathrm{~mL})$ in hexane $(1.6 \mathrm{M}$, $12.8 \mathrm{mmol}$ ) at $0{ }^{\circ} \mathrm{C}$ under $\mathrm{N}_{2}$. The mixture was stirred for $1 \mathrm{~h}$ at $0{ }^{\circ} \mathrm{C}$. The mixture was then cooled at $-78{ }^{\circ} \mathrm{C}$, and DMF $(2.3 \mathrm{~mL}, 29 \mathrm{mmol})$ was added. After $2 \mathrm{~h}$ at $-78{ }^{\circ} \mathrm{C}$, sat. $\mathrm{NH}_{4} \mathrm{Cl}$ aq. was added to quench the reaction. The crude product was extracted with $\mathrm{Et}_{2} \mathrm{O}$. The organic layer was dried over $\mathrm{MgSO}_{4}$ and concentrated to give a crude product, which was purified by flash column chromatography to afford the $o$-formylated MOM ether $(0.98 \mathrm{~g}, 76 \%)$.

To the $o$-formylated MOM ether $(0.98 \mathrm{~g}, 5.5 \mathrm{mmol})$ in THF $(7 \mathrm{~mL})$ was added $2 \mathrm{~N} \mathrm{HCl}$ aq. $(2 \mathrm{~mL})$ at $\mathrm{rt}$. The resulting mixture was heated at reflux $\left(70{ }^{\circ} \mathrm{C}\right)$ for $4 \mathrm{~h}$. After it was cooled at $\mathrm{rt}, \mathrm{H}_{2} \mathrm{O}$ (ca. $20 \mathrm{~mL}$ ) was added, and the crude salicylaldehyde derivative was extracted with $\mathrm{Et}_{2} \mathrm{O}$. Purification by flash column chromatography afforded 2-hydroxy-3-methylbenzaldehyde $(0.62 \mathrm{~g}, 84 \%)$.

\section{Preparation of the catalyst (entry 12, Table 1).}

To a solution of $N$-(2-hydroxy-3,5-dimethylbenzylidene)-2,4,6-tri-tert-butylaniline (15.7 mg, $0.040 \mathrm{mmol})$ in toluene $(0.20 \mathrm{~mL})$ was added $0.10 \mathrm{M} \mathrm{Et}_{3} \mathrm{Al}(0.20 \mathrm{~mL}, 0.020 \mathrm{mmol})$ under $\mathrm{N}_{2}$ at rt. The clear solution was heated at $70{ }^{\circ} \mathrm{C}$ for $1 \mathrm{~h}$. The obtained catalyst 1 was used without purification.

\section{Polymerization (entry 12, Table 1).}

To catalyst 1 in toluene, $0.10 \mathrm{M} \mathrm{BnOH}(0.20 \mathrm{~mL}, 0.020 \mathrm{mmol})$ and then $1.4 \mathrm{~mL}$ of toluene were added at $\mathrm{rt}$, followed by CL $(0.22 \mathrm{~mL}, 1.99 \mathrm{mmol})$. The solution was stirred at $25^{\circ} \mathrm{C}$ for $10 \mathrm{~min}$. Then the reaction mixture was exposed to air to deactivate the catalyst, and a small amount of the reaction mixture was taken to analyze the monomer conversion by ${ }^{1} \mathrm{H}$ NMR (400 $\left.\mathrm{MHz}\right)$ and $M_{\mathrm{n}}$ by SEC. 


\section{Preparation of the complexes for NMR analysis.}

\section{(1) $\mathrm{LAlEt}_{2}$ complex}

To a solution of $N$-(2-hydroxy-3,5-dimethylbenzylidene)-2,4,6-tri-tert-butylaniline (39 mg, $0.099 \mathrm{mmol})$ in toluene- $d_{8}(1.9 \mathrm{~mL})$ was added $0.99 \mathrm{M} \mathrm{Et}_{3} \mathrm{Al}$ in toluene $(0.10 \mathrm{~mL}, 0.099 \mathrm{mmol})$ under $\mathrm{N}_{2}$ at rt. The clear solution was heated at $70{ }^{\circ} \mathrm{C}$ for $1 \mathrm{~h}$ to afford the $\mathrm{LAlEt}_{2}$ complex in toluene- $d_{8}$ and toluene (19/1).

${ }^{1} \mathrm{H}$ NMR (toluene- $\left.d_{8}, 400 \mathrm{MHz}\right): \delta 7.81(\mathrm{~s}, 1 \mathrm{H}, \mathrm{Ar}-\mathrm{N}=\mathrm{CH}-\mathrm{Ar}), 7.57\left(\mathrm{~s}, 2 \mathrm{H}, \mathrm{N}-\mathrm{Ar}-\mathrm{H}_{\text {meta }} \times 2\right.$ ), $6.91(\mathrm{~d}$, $\left.J=2.4 \mathrm{~Hz}, 1 \mathrm{H}, \mathrm{O}-\mathrm{Ar}-\mathrm{H}_{\text {meta }}\right), 6.44\left(\mathrm{~s}, 1 \mathrm{H}, \mathrm{O}-\mathrm{Ar}-\mathrm{H}_{\text {meta }}\right), 2.31\left(\mathrm{~s} 3 \mathrm{H}, \mathrm{Ar}-\mathrm{CH}_{3}\right), 1.99\left(\mathrm{~s}, 3 \mathrm{H}, \mathrm{Ar}-\mathrm{CH}_{3}\right)$, $1.41(\mathrm{~s} 18 \mathrm{H}, \mathrm{tBu} \times 2), 1.29\left(\mathrm{t}, J=8.0 \mathrm{~Hz}, 6 \mathrm{H}, \mathrm{AlCH}_{2} \underline{\mathrm{CH}}_{3} \times 2\right), 1.29(\mathrm{~s}, 9 \mathrm{H}, \mathrm{tBu}), 0.42 \& 0.34(\mathrm{dq}, J=$ 14.6, 8.2 Hz, $\left.2 \mathrm{H}, \mathrm{AlC} \underline{H}_{2} \mathrm{Me}\right)$.

${ }^{13} \mathrm{C}$ NMR $\left(\mathrm{C}_{6} \mathrm{D}_{6}, 100 \mathrm{MHz}\right): \delta 176.9(\mathrm{Ar}-\mathrm{CH}=\mathrm{N}-), 162.5,148.4,143.9,143.7$ (aromatic quaternary carbons), $140.0\left(\mathrm{OAr}_{\text {meta }}\right), 131.5\left(\mathrm{OAr}_{\text {meta }}\right), 131.0,126.4$ (aromatic quaternary carbons), $124.9\left(\mathrm{NAr}_{\text {meta }} \mathrm{X}\right.$ 2), 118.8 (an aromatic quaternary carbon), $37.5\left(\mathrm{NAr}_{\text {ortho }}-\mathrm{CMe}_{3} \times 2\right), 34.8\left(\mathrm{NAr}_{\text {para }}-\mathrm{CMe}_{3}\right), 34.4$ $\left(\mathrm{NAr}_{\text {ortho }}-\mathrm{CMe}_{3}\right), 31.3\left(\mathrm{NAr}_{\text {para }}-\mathrm{CMe}_{3}\right), 20.2\left(\mathrm{Ar}-\mathrm{CH}_{3}\right), 16.3\left(\mathrm{Ar}-\mathrm{CH}_{3}\right), 9.8\left(\mathrm{Ar}-\mathrm{CH}_{3}\right), 2.4\left(\mathrm{Ar}-\mathrm{CH}_{3}\right)$.

(2) $\mathrm{L}_{2}$ AlEt complex (complex 1)

To a solution of $N$-(2-hydroxy-3,5-dimethylbenzylidene)-2,4,6-tri-tert-butylaniline (78 mg, $0.20 \mathrm{mmol})$ in toluene- $d_{8}(1.9 \mathrm{~mL})$ was added $0.99 \mathrm{M} \mathrm{Et}_{3} \mathrm{Al}$ in toluene $(0.10 \mathrm{~mL}, 0.099 \mathrm{mmol})$ under $\mathrm{N}_{2}$ at rt. The clear solution was heated at $70{ }^{\circ} \mathrm{C}$ for $1 \mathrm{~h}$ to afford the $\mathrm{L}_{2} \mathrm{AlEt}$ complex in toluene- $d_{8}$ and toluene (19/1).

${ }^{1} \mathrm{H}$ NMR (toluene- $d_{8}, 400 \mathrm{MHz}$ ): $\delta 9.14(\mathrm{~s}, 1 \mathrm{H}, \mathrm{Ar}-\mathrm{N}=\mathrm{CH}-\mathrm{Ar}), 8.21$ (d, $J=2.0 \mathrm{~Hz}, 1 \mathrm{H}, \mathrm{OAr}-\mathrm{H}_{\text {meta }}$ ), $8.02(\mathrm{~s}, 1 \mathrm{H}, \mathrm{Ar}-\mathrm{N}=\mathrm{CH}-\mathrm{Ar}), 7.54\left(\mathrm{~m}, 4 \mathrm{H}, \mathrm{NAr}-\mathrm{H}_{\text {meta }}\right), 6.88\left(\mathrm{~d}, J=2.0 \mathrm{~Hz}, 1 \mathrm{H}, \mathrm{OAr}-\mathrm{H}_{\text {meta }}\right), 6.84(\mathrm{~d}, J=$ $2.0 \mathrm{~Hz}, 1 \mathrm{H}, \mathrm{OAr}-\mathrm{H}_{\text {meta }}$ ), $6.44\left(\mathrm{~s}, 1 \mathrm{H}, \mathrm{OAr}-\mathrm{H}_{\text {meta }}\right.$ ), 2.12 (overlapping on toluene peak; s, 3H, $\mathrm{Ar}-\mathrm{CH}_{3}$ ), $1.92\left(\mathrm{~s}, 3 \mathrm{H}, \mathrm{Ar}-\mathrm{CH}_{3}\right), 1.82\left(\mathrm{~s}, 3 \mathrm{H}, \mathrm{Ar}-\mathrm{CH}_{3}\right), 1.62,(\mathrm{~s}, 9 \mathrm{H}, \mathrm{tBu}), 1.52\left(\mathrm{~s}, 3 \mathrm{H}, \mathrm{Ar}-\mathrm{CH}_{3}\right), 1.50(\mathrm{~s}, 9 \mathrm{H}, \mathrm{tBu})$, $1.48(\mathrm{~s}, 9 \mathrm{H}, \mathrm{tBu}), 1.38(\mathrm{~s}, 9 \mathrm{H}, \mathrm{tBu}), 1.32(\mathrm{~s}, 9 \mathrm{H}, \mathrm{tBu}), 1.290(\mathrm{~s}, 9 \mathrm{H}, \mathrm{tBu}), 1.09(\mathrm{t}, J=8.2 \mathrm{~Hz}, 3 \mathrm{H}$, $\left.\mathrm{AlCH}_{2} \underline{\mathrm{C}}_{3}\right), 0.28\left(\mathrm{dq}, J=14.6,8.2 \mathrm{~Hz}, 1 \mathrm{H}, \mathrm{Al}-\mathrm{C}_{\underline{H}} \mathrm{HCH}_{3}\right), 0.25(\mathrm{dq}, J=14.6,8.2 \mathrm{~Hz}, 1 \mathrm{H}$, $\mathrm{Al}-\mathrm{CH} \underline{\mathrm{HCH}})_{3}$.

${ }^{13} \mathrm{C}$ NMR $\left(\mathrm{C}_{6} \mathrm{D}_{6}, 100 \mathrm{MHz}\right): \delta 176.5$ (Ar-CH=NAr), 161.9 (quaternary C), 160.5 (Ar-CH=NAr), 156.8 (quaternary C), 152.9 (quaternary C), 148.7 (quaternary C), 144.1 (quaternary C), 143.2 (quaternary C), 142.0 (quaternary $\mathrm{C}$ ), $140.6\left(\mathrm{OAr}_{\text {meta }}\right), 140.2$ (quaternary $\mathrm{C}$ ), 139.5 (quaternary $\mathrm{C}$ ), 139.1 (quaternary $\mathrm{C}$ ), 135.1 ( $\left.\mathrm{OAr}_{\text {meta }}\right), 131.3$ ( $\left.\mathrm{OAr}_{\text {meta }}\right), 130.0$ (quaternary $\mathrm{C}$ ), 129.3 (quaternary $\mathrm{C}$ ), 128.5 (quaternary $\mathrm{C}$ ), $127.3\left(\mathrm{NAr}_{\text {meta }}\right), 127.2\left(\mathrm{NAr}_{\text {meta }}\right), 125.8\left(\mathrm{OAr}_{\text {meta }}\right.$ or quaternary $\left.\mathrm{C}\right), 125.7$ (quaternary $\mathrm{C}$ or $\left.\mathrm{OAr}_{\text {meta }}\right)$, 125.2 (quaternary $\mathrm{C}), 121.7\left(\mathrm{NAr}_{\text {meta }}\right), 121.6\left(\mathrm{NAr}_{\text {meta }}\right), 117.1$ (quaternary $\left.\mathrm{C}\right), 38.8\left(\mathrm{NAr}-\mathrm{CMe}_{3}\right), 38.4$ $\left(\mathrm{NAr}-\mathrm{CMe}_{3}\right), 36.4\left(\mathrm{NAr}-\mathrm{CMe}_{3}\right), 36.3\left(\mathrm{NAr}-\mathrm{CMe}_{3}\right), 35.7\left(\mathrm{NAr}-\mathrm{C}\left(\underline{\mathrm{C}} \mathrm{H}_{3}\right)_{3}\right), 35.3\left(\mathrm{NAr}-\mathrm{C}\left(\underline{\mathrm{C}} \mathrm{H}_{3}\right)_{3}\right), 34.9$ $\left(\mathrm{NAr}-\mathrm{CMe}_{3}\right), 34.7\left(\mathrm{NAr}-\mathrm{CMe}_{3}\right), 32.4\left(\mathrm{NAr}-\mathrm{C}\left(\underline{\mathrm{C}} \mathrm{H}_{3}\right)_{3}\right), 32.1\left(\mathrm{NAr}-\mathrm{C}\left(\underline{\mathrm{C}} \mathrm{H}_{3}\right)_{3}\right), 32.0\left(\mathrm{NAr}-\mathrm{C}\left(\underline{\mathrm{C}} \mathrm{H}_{3}\right)_{3}\right), 31.3$ $\left(\mathrm{NAr}-\mathrm{C}\left(\underline{\mathrm{CH}}_{3}\right)_{3}\right), 20.8\left(\mathrm{ArCH}_{3}\right), 20.0\left(\mathrm{ArCH}_{3}\right), 16.1\left(\mathrm{ArCH}_{3}\right), 15.8\left(\mathrm{ArCH}_{3}\right), 8.9\left(\mathrm{AlCH}_{2} \underline{\mathrm{CH}_{3}}\right), 2.2$ $\left(\mathrm{Al} \underline{\mathrm{CH}} \mathrm{H}_{2} \mathrm{Me}\right)$. 
${ }^{1} \mathrm{H}$ NMR (400 MHz) of $\mathrm{LAlEt}_{2}$ (toluene- $d_{8}$ )

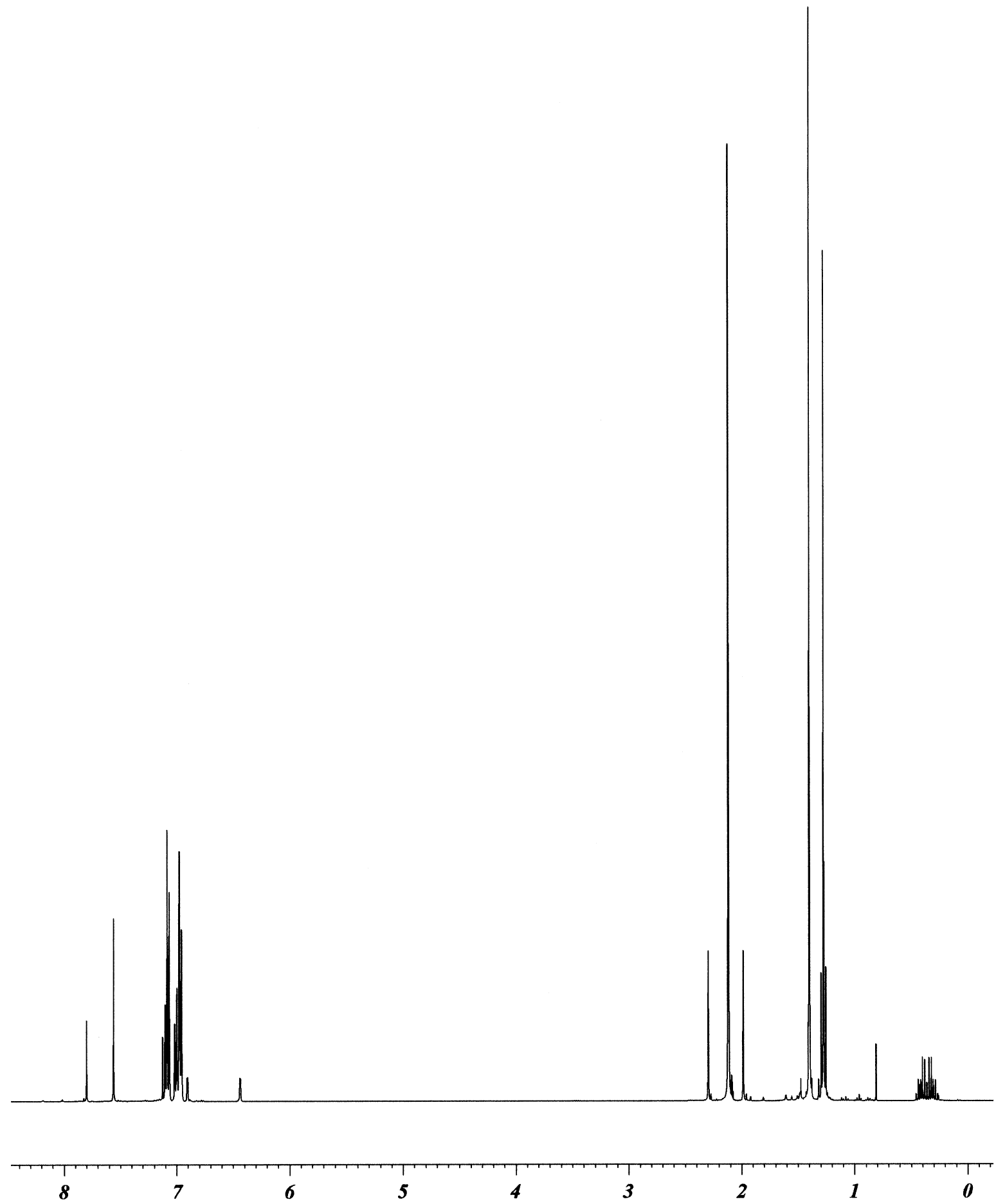


${ }^{13} \mathrm{C}$ NMR $(100 \mathrm{MHz})$ of $\mathrm{LAlEt}_{2}\left(\mathrm{C}_{6} \mathrm{D}_{6}\right)$
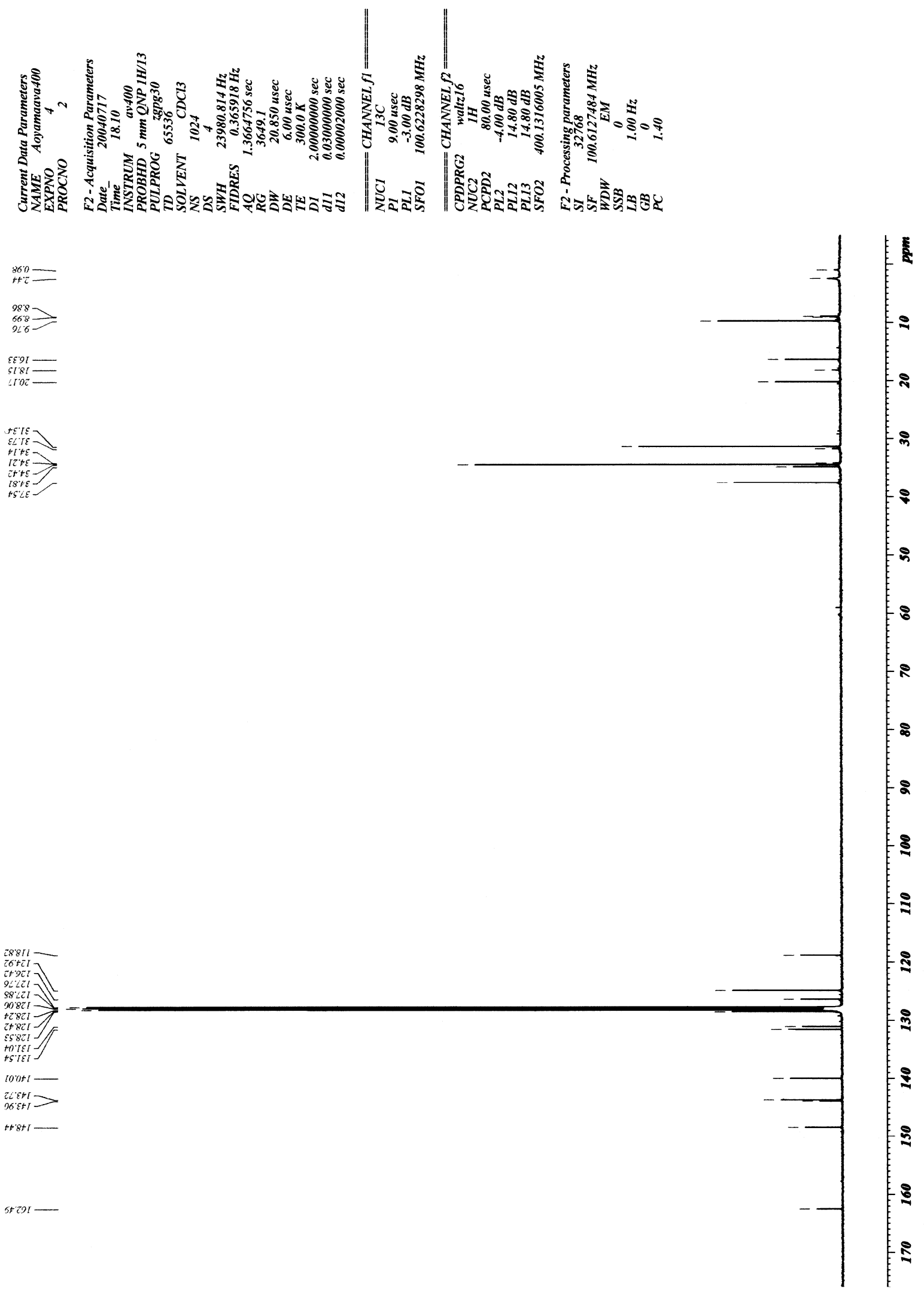


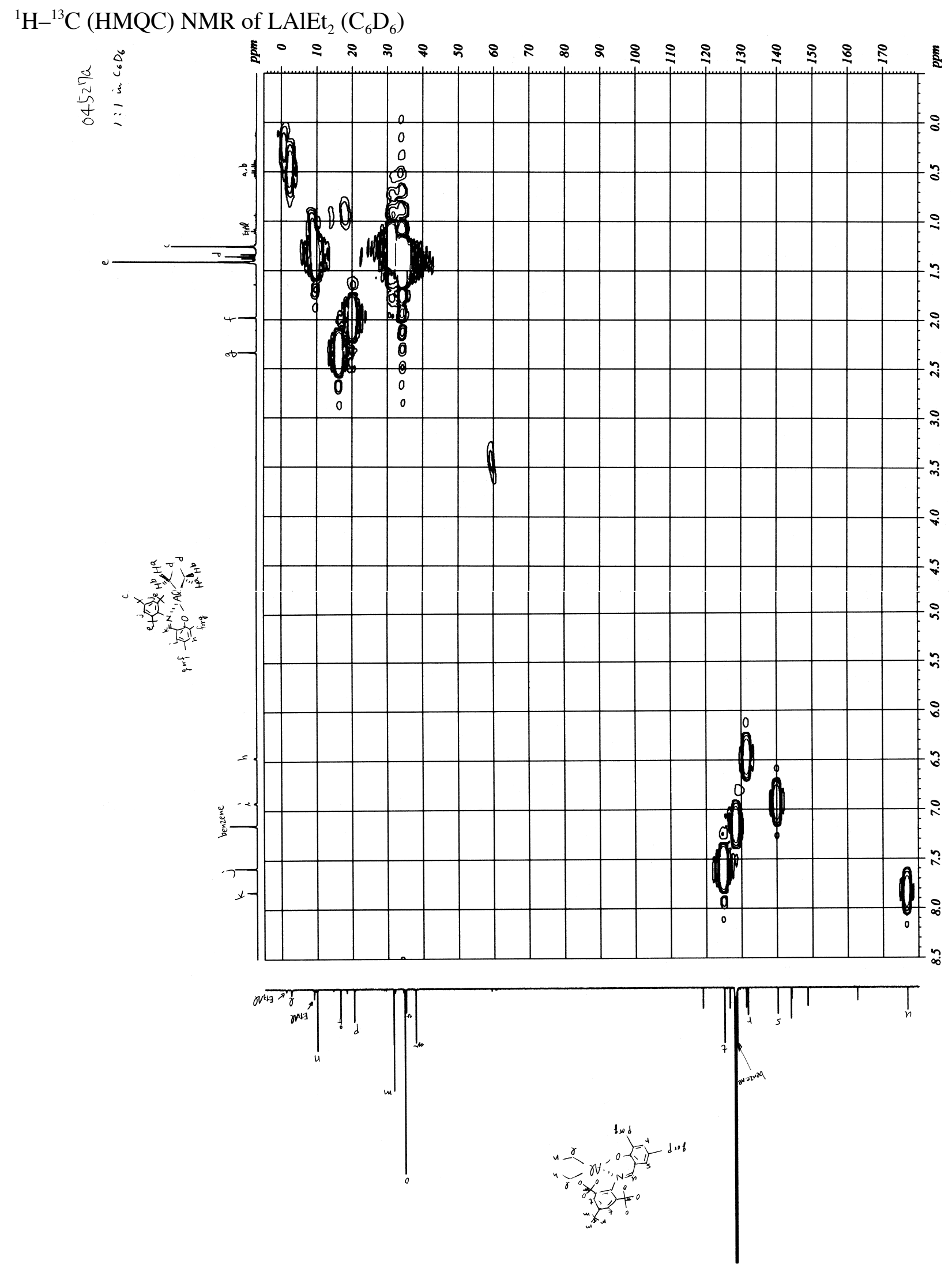


${ }^{1} \mathrm{H}$ NMR (400 MHz) of $\mathrm{L}_{2}$ AlEt (toluene- $d_{8}$ )

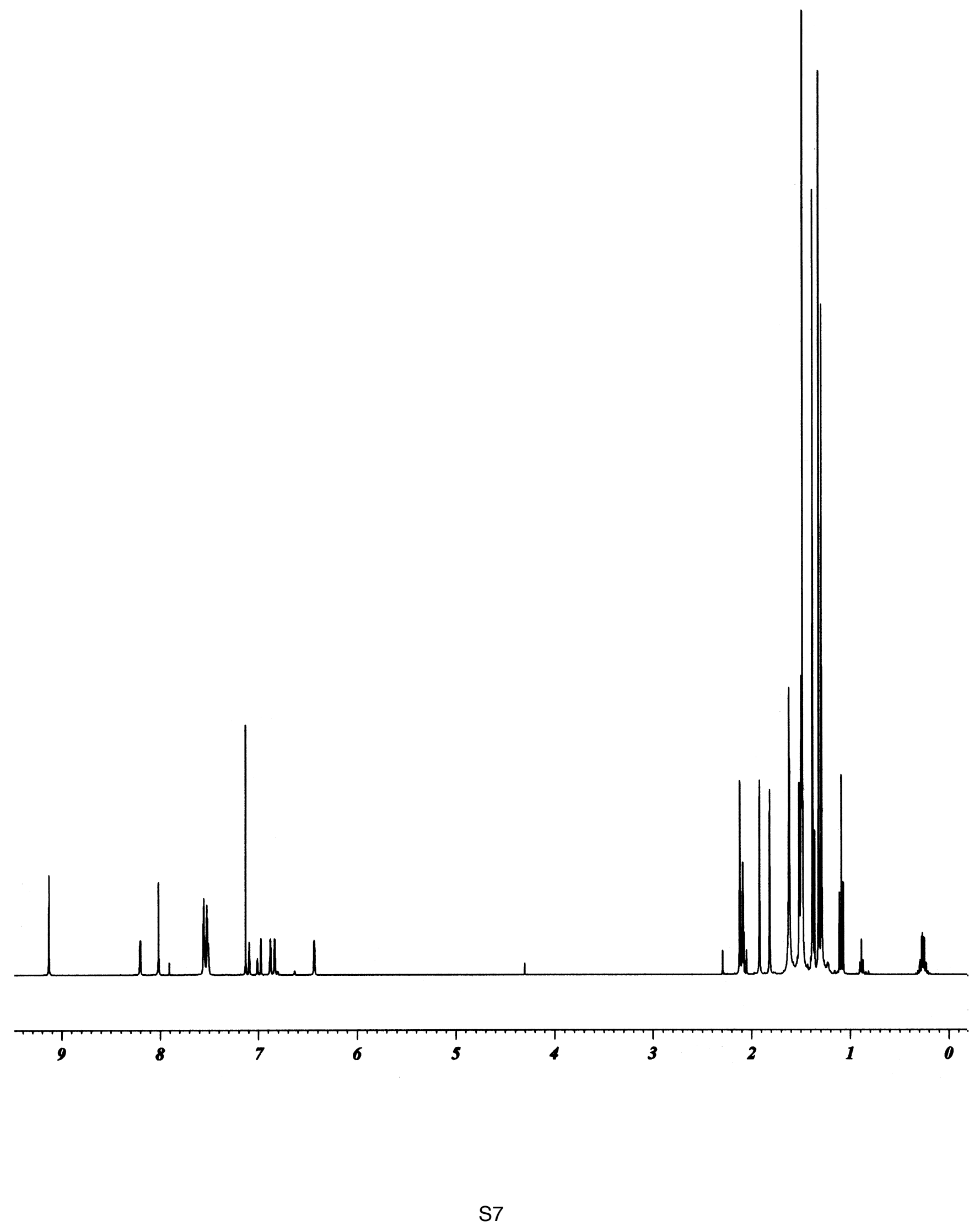


${ }^{13} \mathrm{C}$ NMR $(100 \mathrm{MHz})$ of $\mathrm{L}_{2} \mathrm{AlEt}\left(\mathrm{C}_{6} \mathrm{D}_{6}\right)$
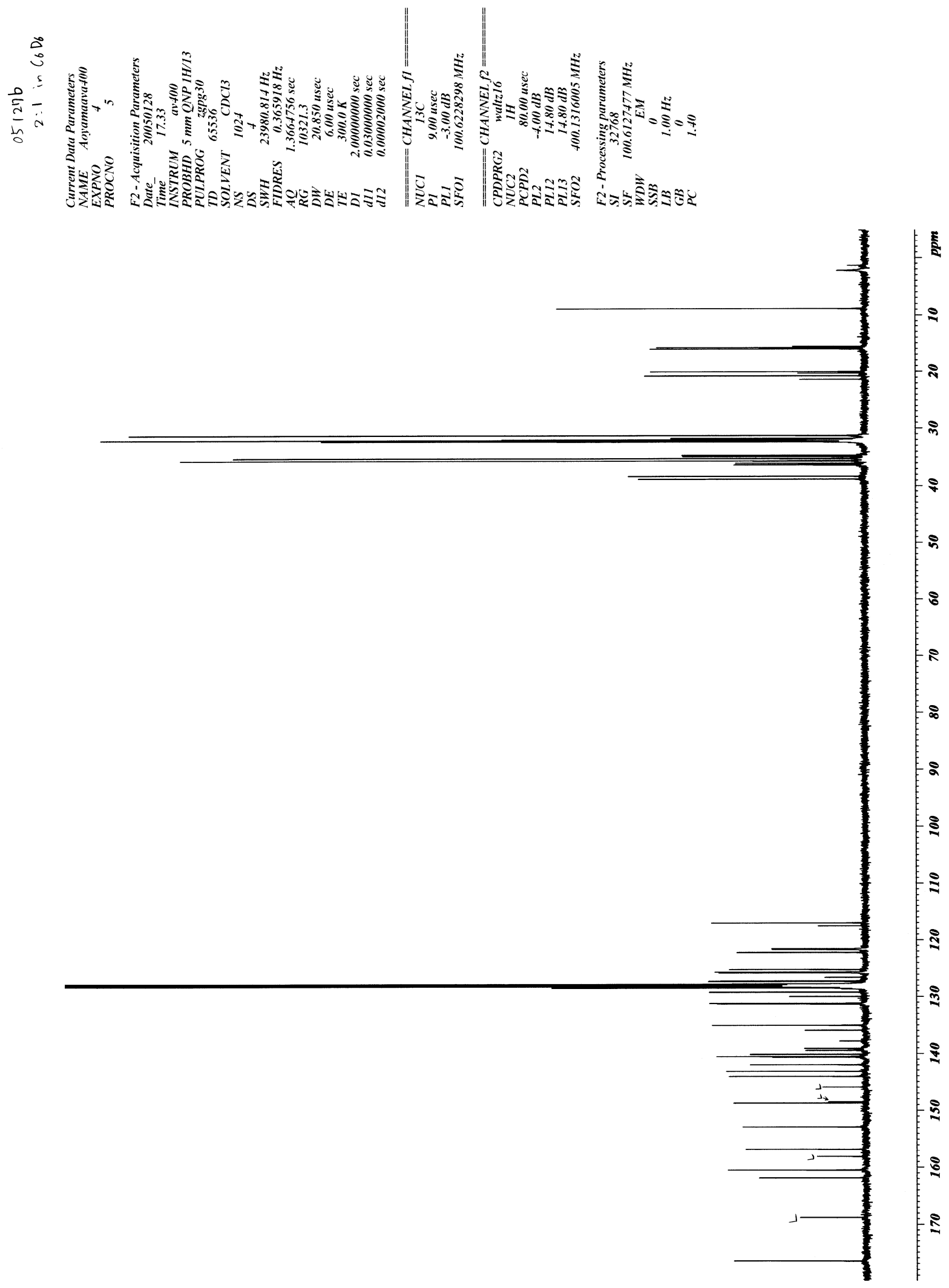
${ }^{1} \mathrm{H}-{ }^{13} \mathrm{C}(\mathrm{HMQC}) \mathrm{NMR}$ of $\mathrm{L}_{2} \mathrm{AlEt}\left(\mathrm{C}_{6} \mathrm{D}_{6}\right)$

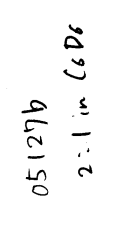

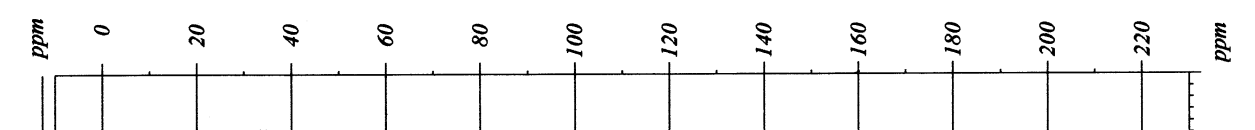
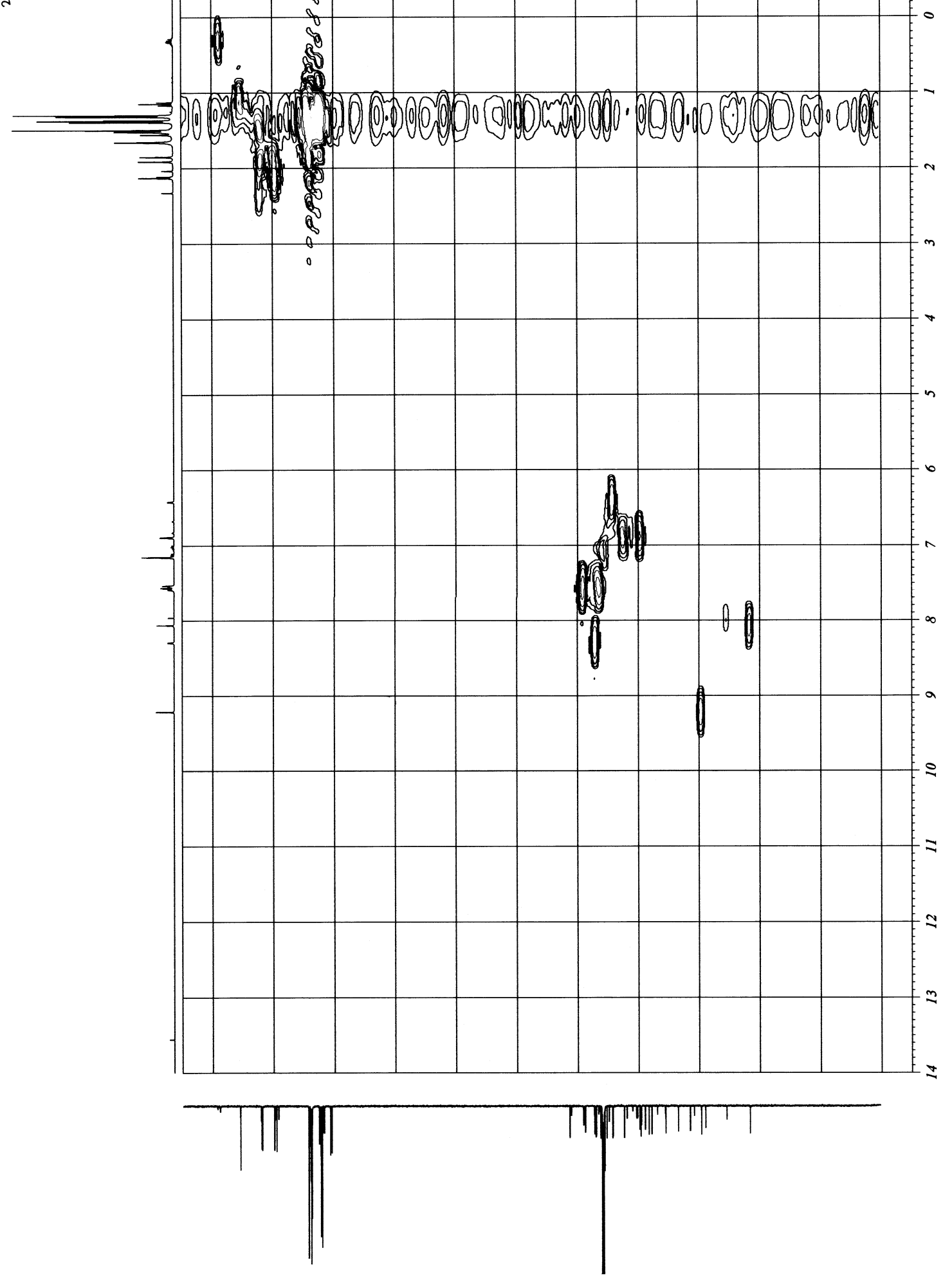
${ }^{27} \mathrm{Al}$ NMR $(156 \mathrm{MHz})$ (toluene- $d_{8}$ )

Conditions: scans, 28000; acquisition time, $0.5734 \mathrm{sec}$; relaxation time, $1.0 \mathrm{sec}$; pulse width, $4.5 \mu$ sec.

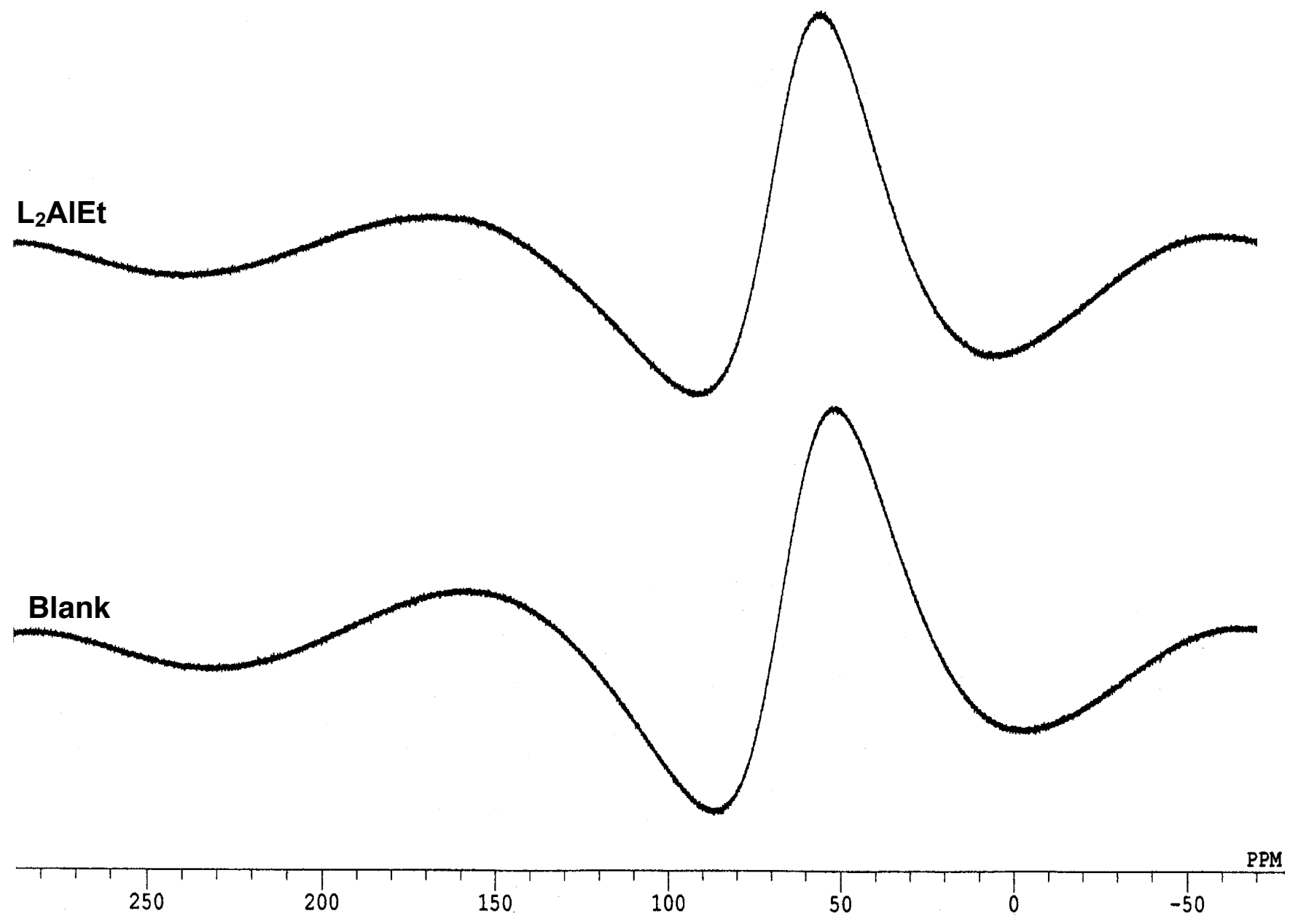


${ }^{1} \mathrm{H}$ NMR (400 MHz, toluene- $d_{8}$ ) of 1 in the presence of 1 equiv. of $\mathrm{BnOH}$ (1)
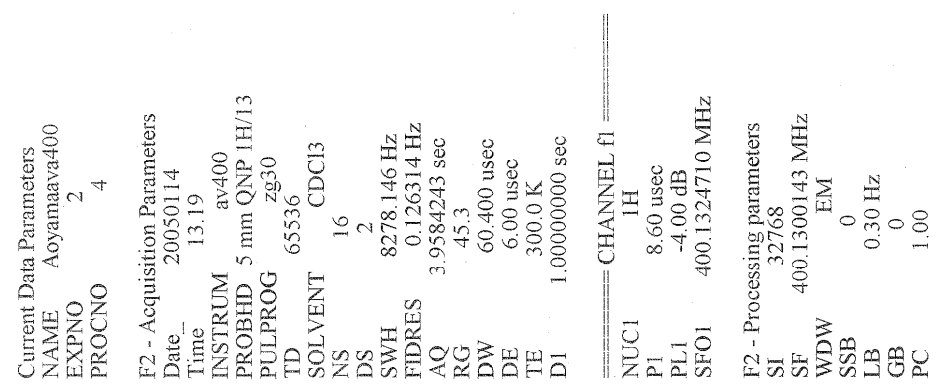

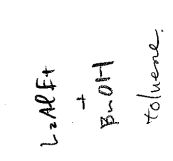
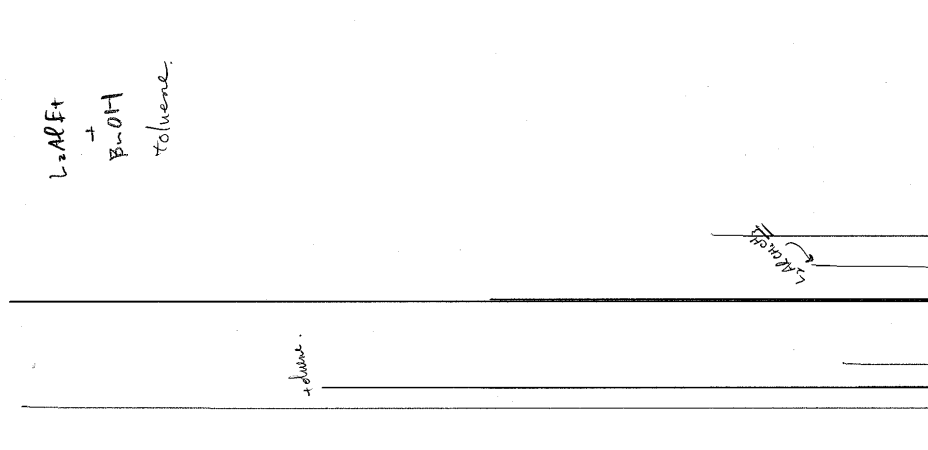
${ }^{1} \mathrm{H}$ NMR (400 MHz, toluene- $d_{8}$ ) of $\mathbf{1}$ in the presence of 1 equiv. of $\mathrm{BnOH}$ (2)
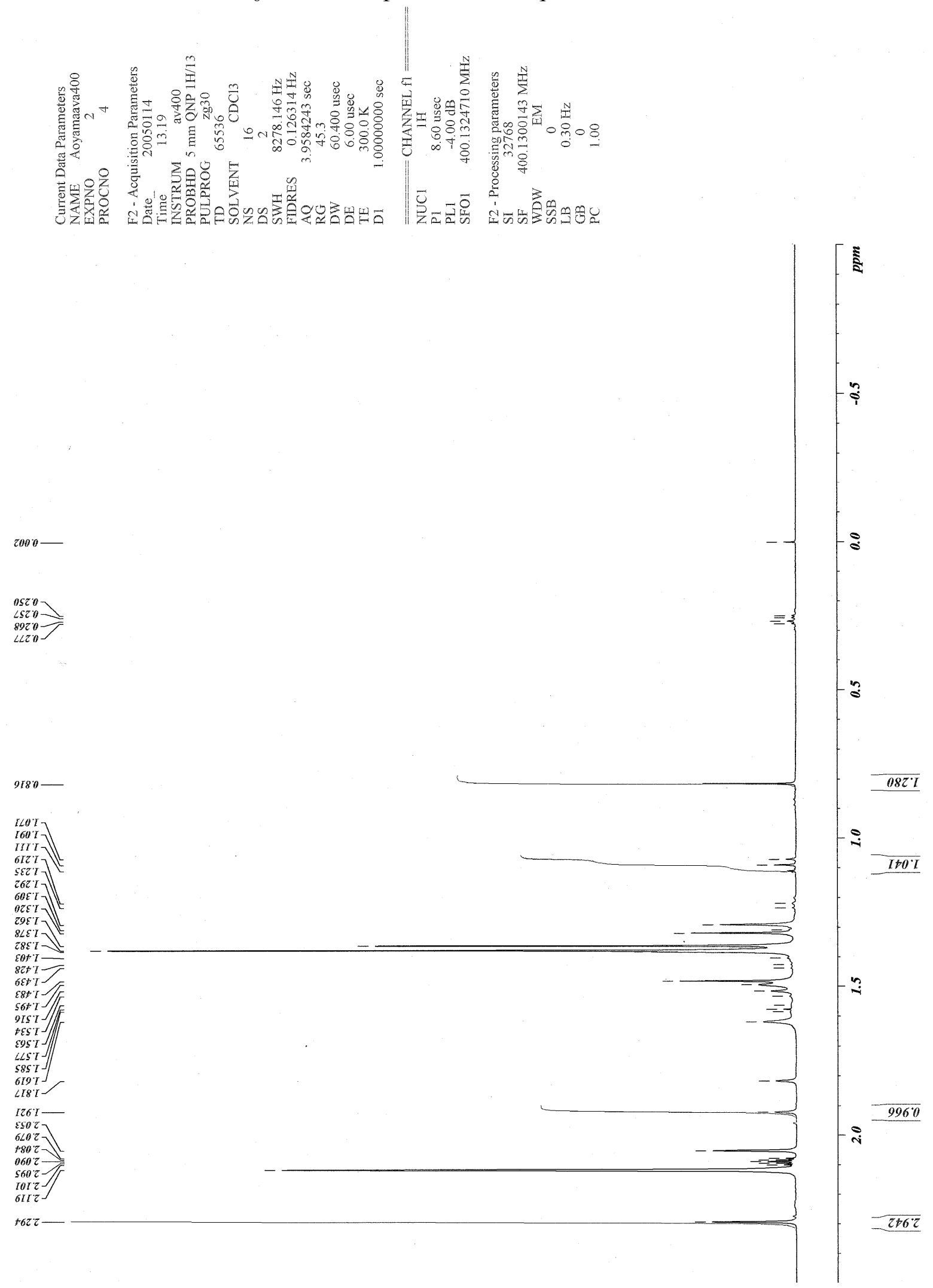
${ }^{1} \mathrm{H}$ NMR (400 MHz, toluene- $d_{8}$ ) of $\mathbf{1}$ in the presence of 1 equiv. of $\mathrm{BnOH}$ (3)
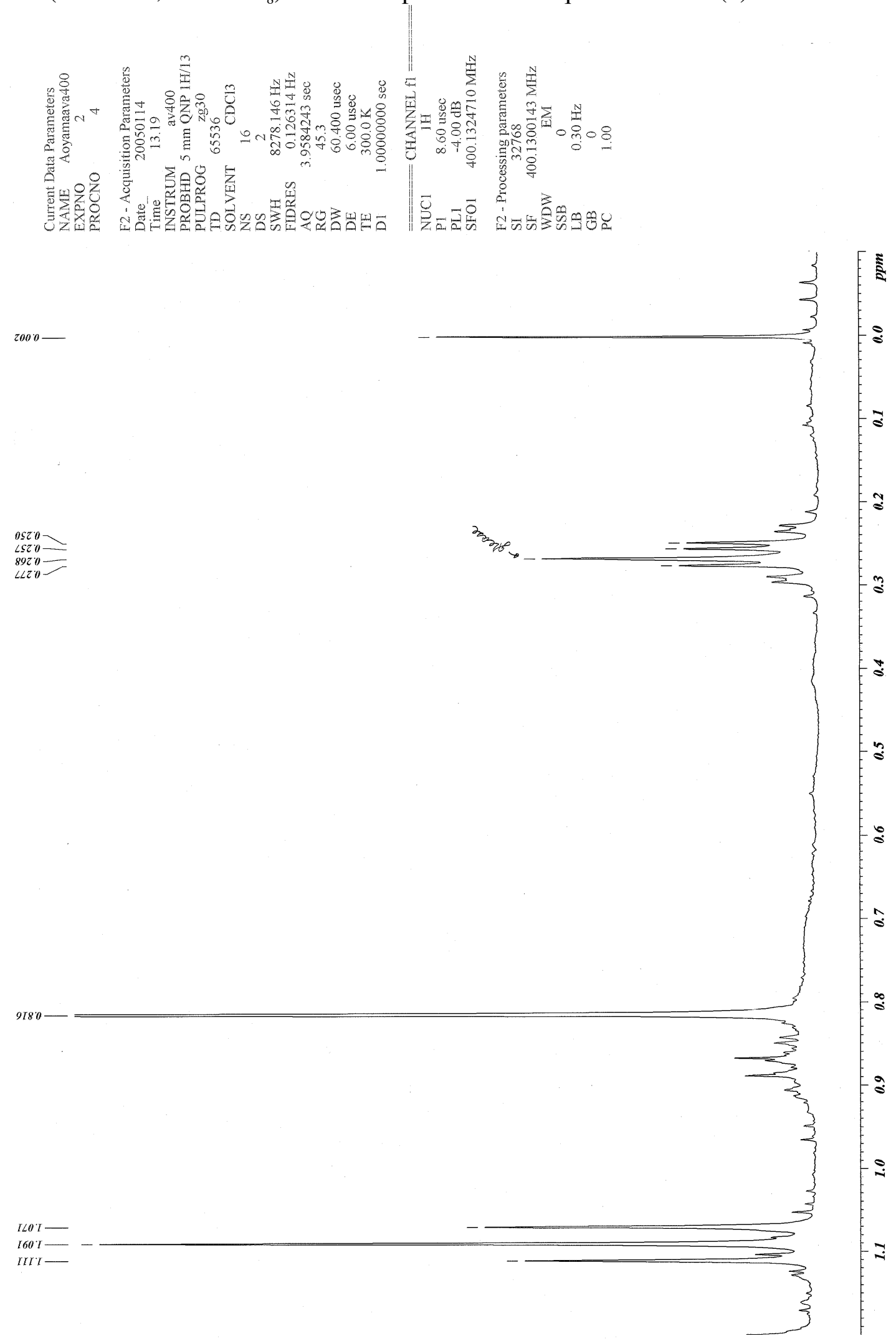
${ }^{1} \mathrm{H}$ NMR (400 MHz, toluene- $d_{8}$ ) of $\mathbf{1}$ in the presence of 1 equiv. of $\mathrm{BnOH}$ (4)
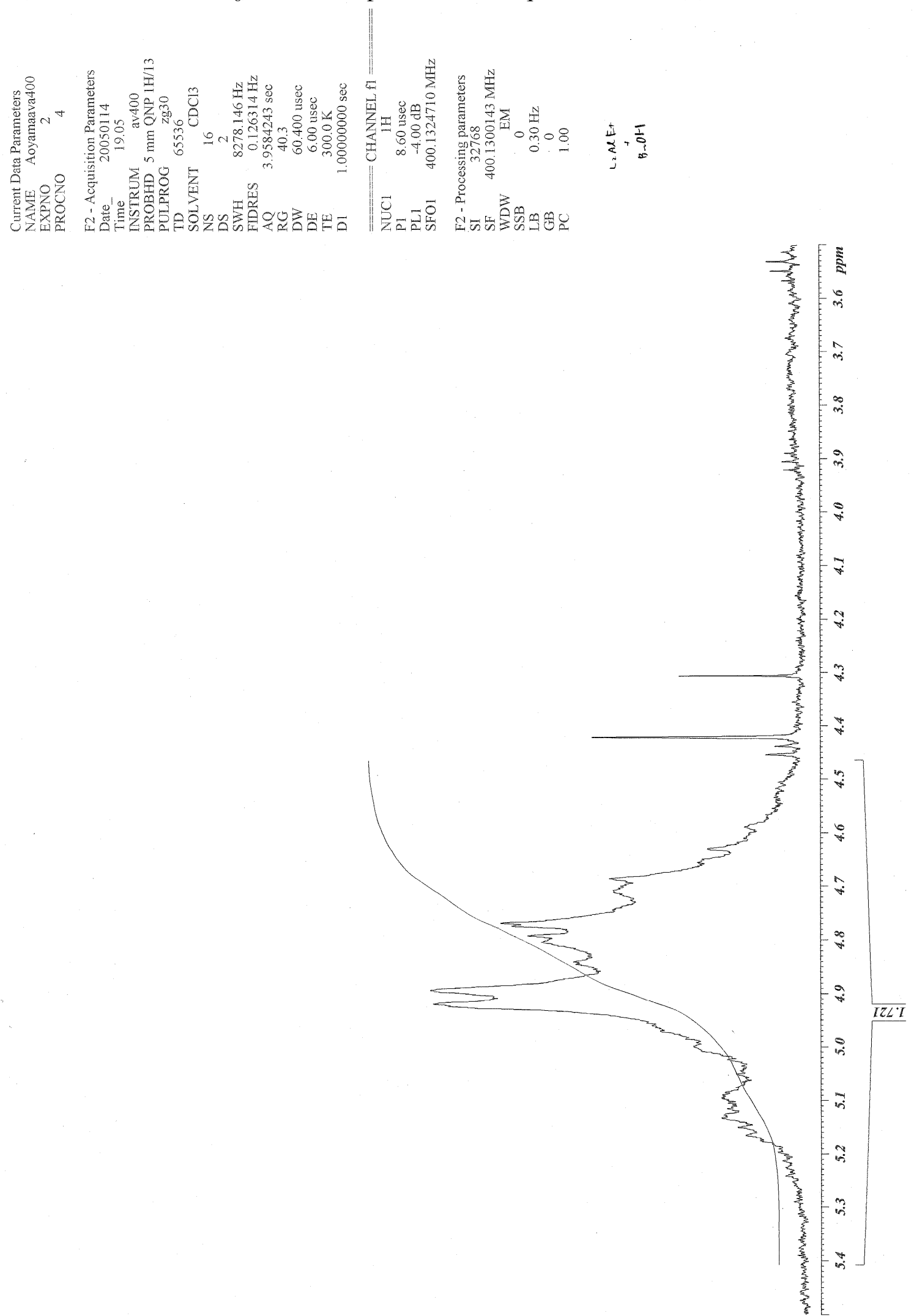
${ }^{1} \mathrm{H}$ NMR (400 MHz, toluene- $d_{8}$ ) of $\mathbf{1}$ in the presence of 1 equiv. of $\mathrm{BnOH}$ (5)
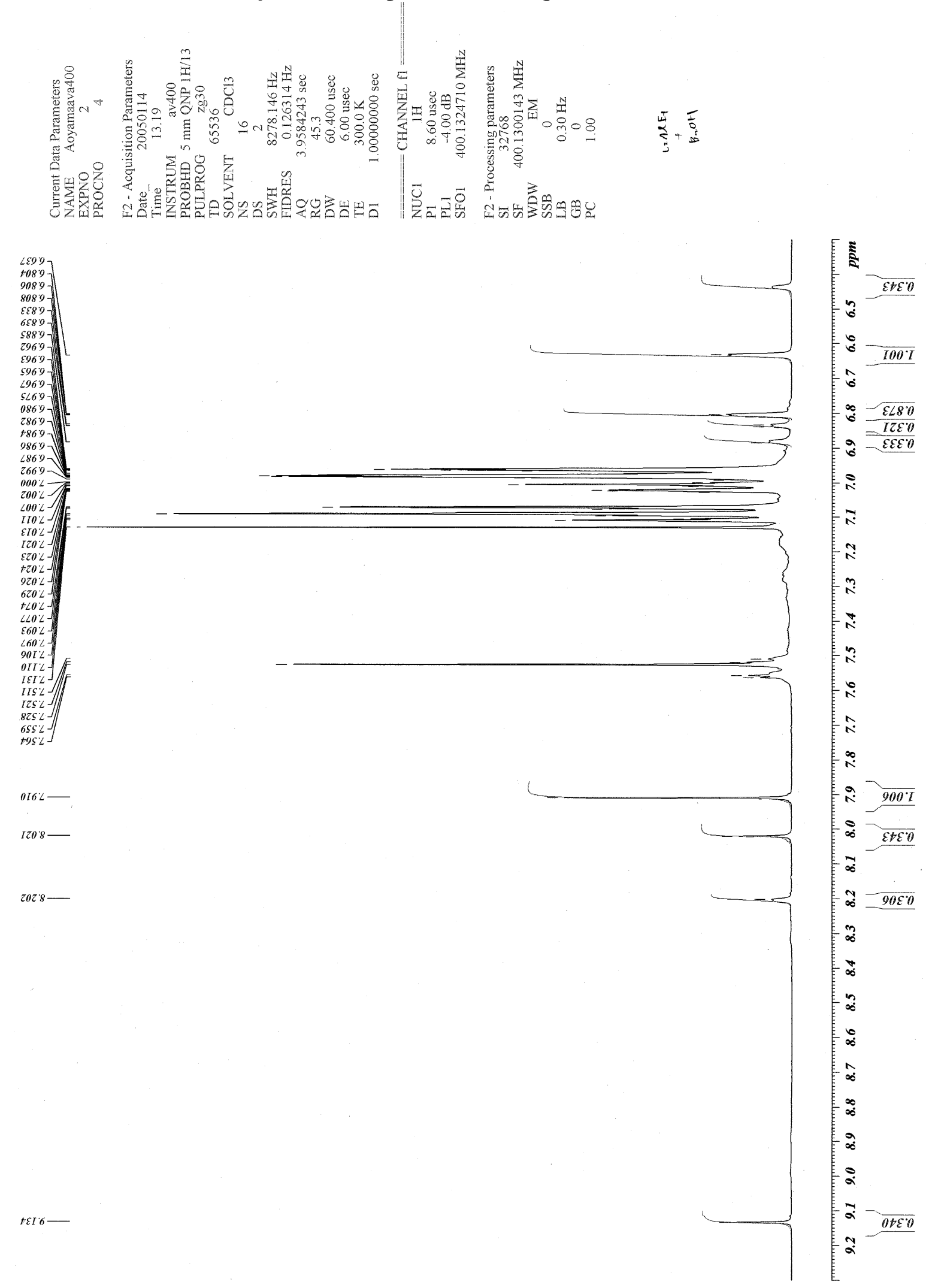